\title{
Endocrinology of aging: the convergence of reductionist science with systems biology and integrative medicine
}

\section{Michael O. Thorner*}

David C. Harrison Medical Teaching Professor of Internal Medicine, Division of Endocrinology and Metabolism, Department of Medicine, University of Virginia, Charlottesville, VA, USA

*Correspondence: mot@virginia.edu

The Endocrinology of Aging is an expanding field. It encompasses the impact of aging on the complex network of endocrine control of the body. The field is on the verge of a fundamental understanding of the factors which appear to either slow or accelerate the process.

Aging is an inevitable process which ultimately leads to a slowing of the bodily processes, less immune surveillance and reduced immunity to disease, reduction in muscle and bone mass which results in frailty which is paralleled with reduced memory and intellectual function. Aging was for a long tome considered to be just an obvious consequence of accumulated wear-and-tear, a passive decline in function caused by a build up of tissue damage that occurred with passing time. This view has dramatically changed due to recent evidence that has established that aging is a regulated process. Genetic studies have clearly indicated that hormonal pathways are central to this regulation of aging and that aging is modifiable to a surprising extent. This has brought endocrinology to the fore in aging research.

A dramatic endocrine change of aging is the development of the menopause which occurs quite suddenly and produces marked symptoms and is associated with major changes in the body which the perimenopausal woman is only too aware of. While menopause is due to the loss of ovarian follicles which can be stimulated it is associated with reduced levels of estradiol and progesterone and increased levels of the gonadotropins and presumably gondotropin releasing hormone. Clearly the impact on the individual is great and probably every system in the body is affected. Is this just due to the reduced levels of gonadal steroids or is this due to secondary changes resulting from the decreased gonadal steroid levels? The large controversy regarding hormone replacement therapy makes clear that there is no consensus as to whether it is advantageous or deleterious to replace gonadal steroids at this stage of life. There is little controversy about replacing them in premenopausal women who undergo surgical oophorectomy.

Aging is a progressive process which begins when function is maximal and then declines from that point. Thus for the hypothalamic/pituitary/growth hormone axis aging appears to start when growth hormone secretion is at its peak, i.e., at mid puberty. From that point on, the secretion of growth hormone declines. However a number of known factors can influence the degree of the decline - the decline appears to be slowed by remaining lean and being physically active with regular exercise. A major question is whether all the changes of aging are due to changes in hormonal state or whether tissues age and thus lose their ability to respond to hormones. My own bias is that there is great plasticity in the system and by having a fundamental understanding of the regulatory processes it will be possible to maintain a youthful hormonal state by life style, nutritional and pharmacological interventions and that this in turn will slow the aging process to extend healthspan rather than lifespan.

Frontiers in Endocrinology of Aging will be the journal which will bring together new knowledge including that generated in multiple models ranging from zebra fish to man, from molecular biology to systems biology and will include not only traditional biological sciences but will also involve the use of biomathematical modeling and in silico science. My own interest in the growth hormone axis prompts me to point out that the growth hormone regulation involves an important interplay of growth hormone with metabolism. Metabolism is exquisitely regulated and this involves not only traditional hormonal systems, but an interaction of neural inputs, autonomic nervous system inputs, circadian rhythms and ultradian rhythms as well as autocrine and paracrine influences. Aging has impact on all these systems.

Our challenge will be integrate reductionist science with systems biology. It is my hope that this journal will broaden the field and be all inclusive and bring the field to its rightful place. Aging is an ever more important area as the baby boomers now are entering into the later part of their seventh decade of life - many members of this group wish to maintain their health and vitality for as long as possible and also to maintain their independence. I believe the field of the endocrinology of aging is well placed to offer new approaches and advances to address these challenges and that Frontiers in Endocrinology of Aging will play an important part in disseminating new knowledge in this field to advance both science and medicine.

Received: 16 September 2010; accepted: 16 September 2010; published online: 14 October 2010.

Citation: Thorner MO (2010) Endocrinology of aging: the convergence of reductionist science with systems biology and integrative medicine. Front. Endocrin. 1:2. doi: 10.3389/ fendo.2010.00002

This article was submitted to Frontiers in Endocrinology of Aging, a specialty of Frontiers in Endocrinology. Copyright (c) 2010 Thorner. This is an open-access article subject to an exclusive license agreement between the authors and the Frontiers Research Foundation, which permits unrestricted use, distribution, and reproduction in any medium, provided the original authors and source are credited. 